\title{
Determinação de Concentrações de Beauveria bassiana (Bals.) Vuill. para o Controle de Insetos-Pragas de Grãos Armazenados
}

\author{
Alcides Moino Jr. ${ }^{1}$ e Sérgio B. Alves ${ }^{1}$ \\ ${ }^{1}$ ESALQ/USP, Departamento de Entomologia, Caixa postal 9, 13418-900, Piracicaba/SP.
}

An. Soc. Entomol. Brasil (26) 1: 15-20 (1997)

Determination of Dosages of Beauveria bassiana (Bals.) Vuill. for Stored Grain Pests Control

\begin{abstract}
The susceptibility of two isolates of Beauveria bassiana (Bals.) Vuill. against Sitophilus oryzae (L.), S. zeamais (Motsch.) and Rhyzopertha dominica (Fabr.) was determined. Boxes containing stored rice previously inoculated with conidia of two B. bassiana isolates were infested with the three insect species and maintained at $26 \pm 0.5^{\circ} \mathrm{C}, 70 \pm 10 \%$ of $\mathrm{RH}$, and photophase of 12 hours. Ali three species were susceptible to both fungal isolates. Both isolates were efficient against $S$. oryzae, at the concentration of $0.5 \mathrm{~g}$ conidia/ $100 \mathrm{~g}$ of grain. The isolate 604 was the most efficient against S. zeamais, at the concentration of $0.1 \mathrm{~g}$ conidia/100 $\mathrm{g}$ of grain. Dosages of $0.01 \mathrm{~g}$ (isolate 476) and $0.05 \mathrm{~g}$ (isolate 604$) / 100 \mathrm{~g}$ of grain provided high mortality levels of $R$. dominica, confirming the higher susceptibility of this species to the pathogen.
\end{abstract}

KEY WORDS: Insecta, stored grain pests, entomopathogenic fungi, microbial control.

RESUMO - Determinou-se a suscetibilidade de Sitophilus oryzae (L.), S. zeamais (Motsch.) e Rhyzopertha dominica (Fabr.) a dois isolados do fungo Beauveria bassiana (Bals.) Vuill. Para isso foram usados recipientes contendo arroz beneficiado, inoculado previamente com conídios dos isolados, infestados com as três espécies de insetos. Esses recipientes foram mantidos a $26 \pm 0,5^{\circ} \mathrm{C}, 70 \pm$ $10 \%$ de UR e fotofase de 12 horas. As três espécies foram suscetíveis aos dois isolados do patógeno. Os dois isolados foram eficientes para o controle de $S$. oryzae, na concentração de $0,5 \mathrm{~g}$ de conídios/100 g de grãos. Para S. zeamais, o isolado 604 foi mais eficiente na concentração de $0,1 \mathrm{~g}$ de conídios/100 g de grãos. Com relação a $R$. dominica, foram suficientes dosagens de $0,01 \mathrm{~g}$ (isolado 476) e $0,05 \mathrm{~g}$ (isolado 604)/100 g de grãos, confirmando a maior suscetibilidade desta espécie ao patógeno.

PALAVRAS-CHAVE: Insecta, pragas de grãos armazenados, fungos entomopatogênicos, controle microbiano.

A utilização contínua de inseticidas para o controle das pragas de grãos armazenados vem causando problemas sérios, como o surgimento de resistência das pragas aos pro- dutos utilizados e a presença de resíduos tóxicos em grãos, rações e outros (Pacheco et al. 1990, Sartori et al. 1990). Dessa forma, há a necessidade de pesquisas com outros mé- 
todos de controle, dentre os quais o controle biológico por entomopatógenos, método que mais se assemelha aos produtos químicos, quanto à facilidade de produção, aplicação e eficácia.

A determinação de dosagens ou concentrações de um determinado patógeno para o controle de insetos é importante na escolha da estratégia para sua utilização. Alguns trabalhos têm sido realizados visando o controle de pragas de grãos armazenados por fungos entomopatogênicos. Ferron \& Robert (1975) efetuaram estudos com o caruncho-do-feijão, Acanthoscelides obtectus (Say), expondo-o a diferentes concentrações de conídios de Beauveria bassiana (Bals.) Vuill., B. tenella (Delacroix) Siemaszko, Metarhizium anisopliae (Metsch.) Sorok. e Paecilomyces fumosoroseus (Wize) Vassiljevski, verificando a ocorrência de infecção pelos quatro patógenos. Hluchy \& Samsinakova (1989) estudaram a suscetibilidade de larvas do $3^{\circ}$ ínstar de Galleria mellonella (L.) e adultos de Sitophilus granarius (L.) a B. bassiana, na formulação "Boverosil". B. Frydocva et al. (dados não publicados) constataram que "Boverosil" foi eficiente no controle de $S$. granarius e Oryzaephilus surinamensis (L.). Searle \& Doberski (1984), Ferron (1977), Rodrigues \& Pratissoli (1990), Lourenção et al. (1993) e Adane et al. (1996) também trabalharam com fungos entomopatogênicos para o controle de pragas de grãos armazenados, estudando fatores que afetam o processo de doença nas condições ambientais específicas do armazenamento de grãos, bem como determinando dosagens necessárias para o controle eficiente dessas pragas. Esta pesquisa teve por objetivo determinar as concentrações dos isolados 476 e 604 de B. bassiana a serem aplicadas em grãos armazenados para o controle de Sitophilus oryzae (L.), S. zeamais Mots. e Rhyzopertha dominica (Fabr.).

\section{Material e Métodos}

Os isolados de $B$. bassiana utilizados foram o 476 e o 604 , selecionados de um total de 72 isolados de $B$. bassiana e $M$. anisopliae. Esses isolados foram inoculados em adultos de $S$. oryzae, S. zeamais e R. dominica, em concentrações de 0,001 a $1,0 \mathrm{~g}$ de conídios/ $100 \mathrm{~g}$ de grãos de arroz beneficiado. A produção do inóculo foi feita em arroz pré-cozido pelo método de bandejas (Alves 1986). Foram utilizados recipientes de plástico $(250 \mathrm{ml}), \mathrm{com}$ $100 \mathrm{~g}$ de arroz beneficiado por recipiente, em cinco repetições por tratamento. Posteriormente, os recipientes com o arroz inoculado foram infestados com 20 insetos adultos de cada espécie e mantidos a $26 \pm 0,5^{\circ} \mathrm{C}, 70 \pm 10$ $\%$ U.R. e fotofase de 12 horas, por 10 dias. Foram usadas as concentrações de 0,001 ; 0,$005 ; 0,01 ; 0,05 ; 0,1 ; 0,5$ e 1,0 g de conídios/ $100 \mathrm{~g}$ de grãos para cada espécie de praga e uma testemunha (sem aplicação do fungo).

A avaliação da mortalidade foi feita 10 dias após a inoculação, sendo os insetos mortos lavados com álcool puro e água destilada para a desinfestação superficial e, em seguida, acondicionados individualmente em placas de poliestireno, as quais permaneceram em câmara úmida para a confirmação da mortalidade pelo patógeno. As mortalidades totais foram comparadas por meio da análise de variância e teste de Tukey, e as concentrações letais medianas $\left(\mathrm{CL}_{50}\right)$ obtidas por meio da análise de Probit, com a utilização do programa MOBAE (M.L. Haddad \& R.C.B. Moraes, não publicado).

\section{Resultados e Discussão}

Analisando-se os valores de CL obtidos pelo método de Probit, não foi verificada diferença, com relação a $S$. oryzae entre o isolado 476 e o isolado 604 (Tabela 1). Com relação a $S$. zeamais, houve maior eficiência do isolado 604, a uma concentração menor, provocando a morte do mesmo número de insetos que o causado pelo isolado 476. Para $R$. dominica, foi possível constatar a sua maior sensibilidade aos isolados 476 e 604 quando comparada às outras duas espécies de insetos estudadas, já que usando concentrações da ordem de 10a 100 vezes mais elevadas, é pos- 
Tabela 1. Concentrações letais medianas ( $\mathrm{g}$ conídios/100 g de grãos), intervalo de confiança (IC), coeficiente angular da reta (b) e valores de $\div 2$ obtidos pela análise de Probit para Beuveria bassiana, isolados 476 e 604, sobre as três espécies de insetos.

\begin{tabular}{lcccr}
\hline Inseto/isolado & $\mathrm{CL}_{50}$ & $\mathrm{IC}$ & Valor de b & \multicolumn{1}{c}{$\chi^{2}$} \\
\hline S. oryzae/476 & 0,0678 & $(0,0470 ; 0,0979)$ & 0,96004 & 5,71 \\
S. oryzae/604 & 0,0915 & $(0,0511 ; 0,1638)$ & 0,84160 & 11,22 \\
S. zeamais/476 & 0,0177 & $(0,0120 ; 0,0261)$ & 0,66895 & 3,36 \\
S. zeamais $/ 604$ & 0,0067 & $(0,0039 ; 0,0115)$ & 0,99607 & 9,98 \\
R. dominica/476 & 0,0004 & $(0,0001 ; 0,0023)$ & 0,99967 & 17,98 \\
$R$. dominica/604 & 0,0013 & $(0,0006 ; 0,0028)$ & 0,99882 & 8,37 \\
\hline
\end{tabular}

sível matar o mesmo número de insetos. Também pode-se observar, pela inclinação das retas demortalidade, com valores de 7,8 e 8,3, respectivamente, para os isolados 604 e 476, que esses isolados foram mais virulentos para $R$. dominica que para as outras duas espécies de Sitophilus.

Com relação à determinação da melhor concentração, observou-se, para $S$. oryzae, que as concentrações de 0,5 e 1,0 g de conídios/ $100 \mathrm{~g}$ de grãos, nos dois isolados, causaram maiores mortalidades após 10 dias da inoculação (Tabela 2). Assim, $0,5 \mathrm{~g}$ de conídios/ $100 \mathrm{~g}$ de grãos pode ser considerada como a concentração mínima indicada para o controle desta praga por meio da utilização dos dois isolados de B. bassiana. A concentração que melhor discriminou os dois isolados testados em $S$. zeamais, foi a de $0,1 \mathrm{~g}$ de conídios/ $100 \mathrm{~g}$ de grãos, indicando maior suscetibilidade deste inseto ao fungo B. bassiana, quando comparado com $S$. oryzae. Com relação a $R$. dominica, houve uma melhor discriminação a partir da concentração de $0,01 \mathrm{~g}$ de conídios/ $100 \mathrm{~g}$ de grãos para o isolado 476. Para o isolado 604, foram obtidas maiores mortalidades a partir da concentração de 0,05 $\mathrm{g}$ de conídios /100 g de grãos, sendo este inseto mais suscetível ao fungo que as duas espécies anteriormente mencionadas.

A maioria dos trabalhos realizados visando a determinação de concentrações de fungos entomopatogênicos necessárias para o controle de pragas de grãos armazenados tem como base a metodologia que implica na pulverização de suspensões aquosas dos propágulos do patógeno sobre os insetos (Searle \& Doberski 1984, Hluchy \& Samsinakova 1989), o que dificulta a comparação com os dados obtidos neste trabalho, onde procurouse uma melhor aproximação com o que seria uma aplicação viável do microrganismo no ambiente de armazenamento. Nessa linha, Lourenção et al. (1993) avaliaram a eficiência de $B$. bassiana, $M$. anisopliae e pirimifósmetil quando adicionados a grãos de milho para o controle de $S$. zeamais. Foram estudadas duas concentrações, sendo 1,25 e 2,5 g de arroz (substrato da produção) + fungo por $125 \mathrm{~g}$ de grãos de milho, correspondendo a $1,0 \times 10^{10}$ e $2,0 \times 10^{10}$ conídios $/ 100 \mathrm{~g}$ de grãos, semelhantes àquelas obtidas neste trabalho como eficientes para o controle da praga. Esses autores obtiveram com B. bassiana, 100 e $98,4 \%$ de mortalidade nas duas concentrações, respectivamente, sendo que $M$. anisopoliae promoveu $86,5 \%$ de mortalidade dos insetos na maior concentração. Esses resultados confirmam a maior eficiência de $B$. bassiana em relação a $M$. anisopliae, que caracterizou-se como um patógeno menos agressivo, fato também observado na fase de seleção dos isolados que foram avaliados no presente trabalho. 
Tabela 2. Percentagens médias de mortalidade de adultos de Sitophilus oryzae, Sitophilus zeamais e Rhyzopertha dominica inoculados com os isolados 476 e 604 de Beauveria bassiana nas diferentes concentrações, 10 dias após a inoculação.

\begin{tabular}{|c|c|c|}
\hline Concentração (g conídios/100 g grãos) & Isolado $476^{1}$ & Isolado $604^{1}$ \\
\hline \multicolumn{3}{|c|}{ S. oryzae } \\
\hline Testemunha & 0 a & $0 \mathrm{a}$ \\
\hline 0,001 & $7,0 \pm 2,00 \mathrm{ab}$ & $7,0 \pm 2,55$ a \\
\hline 0,005 & $10,0 \pm 3,53 \mathrm{ab}$ & $17,0 \pm 3,39$ a \\
\hline 0,01 & $22,0 \pm 5,14$ b & $10,0 \pm 1,58$ a \\
\hline 0,05 & $44,0 \pm 8,57 \mathrm{c}$ & $44,0 \pm 1,87 \mathrm{~b}$ \\
\hline 0,1 & $52,0 \pm 2,55 \mathrm{c}$ & $55,0 \pm 6,89 \mathrm{~b}$ \\
\hline 0,5 & $84,0 \pm 2,45 \mathrm{~d}$ & $77,0 \pm 5,38 \mathrm{c}$ \\
\hline 1,0 & $87,0 \pm 2,55 \mathrm{~d}$ & $77,0 \pm 4,35 \mathrm{c}$ \\
\hline \multirow[t]{2}{*}{$\mathrm{CV}$} & $25,52 \%$ & $25,55 \%$ \\
\hline & \multicolumn{2}{|c|}{ S. zeamais } \\
\hline Testemunha & $8,0 \pm 2,55$ a & $8,0 \pm 2,55$ a \\
\hline 0,001 & $26,0 \pm 3,00 \mathrm{ab}$ & $29,0 \pm 7,64 \mathrm{~b}$ \\
\hline 0,005 & $41,0 \pm 7,97 \mathrm{~b}$ & $54,0 \pm 4,30 \mathrm{c}$ \\
\hline 0,01 & $45,0 \pm 7,41 \quad b$ & $56,0 \pm 4,84 \mathrm{c}$ \\
\hline 0,05 & $66,0 \pm 3,67 \mathrm{c}$ & $78,0 \pm 4,63 \mathrm{~d}$ \\
\hline 0,1 & $78,0 \pm 2,00 \mathrm{~cd}$ & $85,0 \pm 0,00 \mathrm{~d}$ \\
\hline 0,5 & $83,0 \pm 2,55 \mathrm{~cd}$ & $99,0 \pm 1,00 \mathrm{e}$ \\
\hline 1,0 & $87,0 \pm 3,39 \mathrm{~d}$ & $100,0 \pm 0,00 \mathrm{e}$ \\
\hline \multirow[t]{2}{*}{$\overline{\mathrm{CV}}$} & $20,40 \%$ & $15,27 \%$ \\
\hline & \multicolumn{2}{|c|}{ R. dominica } \\
\hline Testemunha & $0 \mathrm{a}$ & $0 \mathrm{a}$ \\
\hline 0,001 & $69,0 \pm 4,84$ b & $49,0 \pm 3,31 \mathrm{~b}$ \\
\hline 0.005 & $75,0 \pm 5,24 \mathrm{~b}$ & $68,0 \pm 4,06 \mathrm{c}$ \\
\hline 0,01 & $99,0 \pm 1,00 \mathrm{c}$ & $80,0 \pm 2,23 \mathrm{~d}$ \\
\hline 0,05 & $98,0 \pm 1,22 \mathrm{c}$ & $90,0 \pm 3,53 \mathrm{~d}$ \\
\hline 0,1 & $99,0 \pm 1,00 \mathrm{c}$ & $100,0 \pm 0,00 \mathrm{e}$ \\
\hline 0,5 & $100,0 \pm 0,00 \mathrm{c}$ & $100,0 \pm 0,00 \mathrm{e}$ \\
\hline 1,0 & $100,0 \pm 0,00 \mathrm{c}$ & $100,0 \pm 0,00 \mathrm{e}$ \\
\hline$\overline{\mathrm{CV}}$ & $7,74 \%$ & $7,67 \%$ \\
\hline
\end{tabular}

${ }^{1}$ Médias seguidas por letras distintas diferem entre si pelo teste de Tukey $(\mathrm{P} \leq 0,05)$.

Também visando a utilização de B. bassiana para o controle de $S$. zeamais, Adane et al. (1996) estudaram 10 isolados deste fungo, verificando variações nos níveis de virulência entre os mesmos. Após a seleção de um isolado mais eficiente (190-520), os 
estudos da relação concentração-mortalidade foram realizados com conídios puros, utilizando concentrações de 0,$1 ; 0,5$ e 1,0 g de conídios de B. bassiana por $25 \mathrm{~g}$ de grãos de milho, obtendo $100 \%$ de mortalidade nas três concentrações após 14 dias. Estas concentrações corresponderiam a 0,4; 2,0 e 4,0 g de conídios por $100 \mathrm{~g}$ de grãos, sendo superiores às testadas neste trabalho, onde índices de mortalidade próximos a $100 \%$ foram obtidos com concentrações que variaram de 0,1 a $1,0 \mathrm{~g}$ de conídios por 100 g de grãos, no período de dez dias de avaliação. Esta comparação demonstra a importância de um programa inicial de seleção, através do qual pode-se avaliar várias características do patógeno, tais como virulência, persistência, capacidade de reprodução, as quais contribuem para a obtenção de um isolado eficiente em concentrações mais baixas, viabilizando a aplicação do controle microbiano, inclusive em condições de armazenamento em larga escala. Deve-se ressaltar também, que os isolados selecionados em condições de laboratório, no caso dos grãos armazenados, tendem a manifestar seu potencial de controle de forma bem semelhante no ambiente de armazenamento, onde as condições são bem estáveis, não diferenciando daquelas em que o patógeno foi selecionado.

\section{Agradecimentos}

À Fundação de Amparo à Pesquisa do Estado de São Paulo (FAPESP), pela concessão de bolsa de estudos que tornou possível a realização desta pesquisa.

\section{Literatura Citada}

\section{Adane, K., D. Moore \& S.A. Archer. 1996.} Preliminary studies on the use of Beauveria bassiana to control Sitophilus zeamais (Coleoptera: Curculionidae) in the laboratory. J. Stored Prod. Res. 32: 105-113.
Alves, S.B. 1986. Produção de fungos entomopatogênicos. In: S.B. Alves, (coord.). Controle microbiano de insetos. São Paulo, Ed. Manole, 407 p.

Ferron, P. 1977. Influence of relative humidity on the development of fungal infection caused by Beauveria bassiana in imagines of Acanthoscelides obtectus. Entomophaga 22: 393-396.

Ferron, P. \& P.H. Robert. 1975-Virulence of entomopathogenic fungi for the adults of Acanthoscelides obtectus. J. Invertebr. Pathol. 25: 379-388.

Hluchy, M. \& A. Samsinakova. 1989. Comparative study on the susceptibility of adult Sitophilus granarius (L.) (Coleoptera: Curculionidae) and larval Galleria mellonella (L.) (Lepidoptera: Pyralidae) to the entomogenous fungus Beauveria bassiana (Bals.)Vuill. J. Stor. Prod. Res. 25: 61-64.

Lourenção, A.L., S. Komatsu \& S.B. Alves. 1993. Aplicação de Beauveria bassiana, Metarhizium anisopliae e pirimifósmetil em milho para controle de Sitophilus zeamais. Ecossistema 18: 69-74.

Pacheco, LA., M.R. Sartori \& R.W.D. Taylor. 1990. Levantamento de resistência de insetos-pragas de grãos armazenados à fosfina, no Estado de São Paulo. Coletânea do ITAL 20: 144-154.

Sartori, M.R., I.A. Pacheco, M. Iaderosa \& R.W.D. Taylor. 1990. Ocorrência e especificidade de resistência ao inseticida malation em insetos-pragas de grãos armazenados no Estado de São Paulo. Coletânea do ITAL 20: 194-209. 
Rodrigues, C. \& D. Pratissoli. 1990. Patogenicidade de Beauveria brongniartii (Sacc.) Petch. e Metarhizium anisopliae (Metsch.) Sorok. e seu efeito sobre o gorgulho do milho e caruncho do feijão. An. Soc. Entomol. Brasil 19: 301-306.
Searle, T. \& J. Doberski. 1984. An investigation on the entomogenous fungus Beauveria hassiana as a potential biological control agent for Oryzaephilus surinamensis. J. Stor. Prod. Res. 20: 17-24.

Recebido em 09/02/96. Aceito em 9/12/96. 